\title{
Competitive Hydrogenation and Hydrodeoxygenation of Oxygen-Substituted Aromatics over Rh/Silica: Catechol, Resorcinol and Hydroquinone
}

\author{
Kathleen Kirkwood ${ }^{1}$ · S. David Jackson ${ }^{1}$ (1) \\ Accepted: 9 March 2021 / Published online: 25 March 2021 \\ (c) The Author(s) 2021
}

\begin{abstract}
The competitive hydrogenation and hydrodeoxygenation (HDO) of dihydroxybenzene isomers, catechol (1,2-dihydroxybenzene), resorcinol (1,3-dihydroxybenzene) and hydroquinone (1,4-dihydroxybenzene), was studied in the liquid phase over a $\mathrm{Rh} /$ silica catalyst at $323 \mathrm{~K}$ and 3 barg hydrogen pressure. Under competitive hydrogenation conditions an order of reactivity of ortho > para > meta was observed. Catechol initially inhibited resorcinol and hydroquinone hydrogenation but not HDO suggesting separate sites for hydrogenation and HDO. When resorcinol and hydroquinone were reacted competitively, HDO became the favoured reaction. The data suggested that cyclohexane and cyclohexanone were primary products. At low dihydroxybenzene (DHB) conversion the ratio of HDO products was dependent upon DHB isomer. When all three DHB isomers were reacted together, initially $86 \%$ of the HDO yield came from catechol with the rest from hydroquinone. When resorcinol finally reacted, HDO products were produced first. Reaction of DHB isomers in pairs using deuterium instead of hydrogen revealed changes in kinetic isotope effect (KIE). The presence of competing reactants had a dramatic effect on the energetics of hydrogenation and HDO reactions of individual components, reinforcing the view that hydrogenation and HDO are mechanistically separate. This effect on reaction energetics observed when more than one substrate was present, highlights the limitations of studying one single model compound as a route to understanding the processes required for the upgrading of a true bio-oil feed.
\end{abstract}

Keywords Competitive hydrogenation $\cdot$ Hydrodeoxygenation $\cdot$ Rhodium/silica $\cdot$ Dihydroxybenzene $\cdot$ Deuterium

\section{Introduction}

The focus of this study is on the hydrogenation and hydrodeoxygenation of components of bio-oil derived from lignin, specifically dihydroxybenzenes. When subjected to fast pyrolysis at $673-873 \mathrm{~K}$ in the absence of air, lignocellulosic biomass can be converted into a liquid bio-oil that contains over 300 individual compounds. Many of the compounds generated are oxygenates, however this increased oxygen content is the cause of many of the negative properties of bio-oil such as low heating value, corrosiveness, high viscosity and instability. Typically substituted phenols are used as model compounds for bio-oil HDO [1-4] as this class of

S. David Jackson

david.jackson@glasgow.ac.uk

1 Centre for Catalysis Research, School of Chemistry, University of Glasgow, Glasgow G12 8QQ, Scotland, UK molecules are commonly produced in lignin decomposition [5]. In a previous study [6] the hydrogenation and hydrodeoxygenation (HDO) of catechol, resorcinol and hydroquinone was reported. That study also highlighted that significant HDO was possible at low temperatures $(<343 \mathrm{~K})$. In this study competitive hydrogenation and low temperature HDO was examined. Studies of competitive hydrogenation are still not common [7-10] and studies of competitive HDO even less so [11-13]. Nevertheless, it is important that competitive reactions are considered given that when lignocellulosic biomass is converted into liquid bio-oil it can contain over 300 individual compounds. Separation prior to treatment is unfeasible and so catalytic systems will need to be developed that can operate in a competitive environment. To enable this, we must understand how reactions in a competitive environment affect each other. In recent studies of bio-oil HDO [14, 15] it was found that although the HDO reaction was still effective, there was a significant change in behaviour relative to single model compounds. 
For example, with guaiacol as a single reactant the products were, with most catalysts, cyclohexane derivatives, whereas for guaiacol in bio-oil HDO the product was mostly aromatic in nature. Indeed, in general the hydrogenation functionality was significantly reduced in a competitive environment [14, 15]. Therefore, having already thoroughly studied the single hydrogenation/HDO of the dihydroxybenzene isomers [6] we undertook to examine the competitive reactions in pairs and with all three isomers using hydrogen and deuterium to aid mechanistic understanding.

\section{Experimental}

The catalyst used throughout this study $(2.5 \% \mathrm{Rh} / \mathrm{silica}$, M01078) was supplied and characterised by Johnson Matthey plc. The catalyst was prepared by incipient-wetness using an aqueous rhodium chloride salt. The silica support was supplied by Davison Catalysts. The catalyst was dried overnight at $333 \mathrm{~K}$ and reduced in flowing hydrogen at $473 \mathrm{~K}$ for $2 \mathrm{~h}$ before being cooled and exposed to air. The BET surface area of the catalyst was $321 \mathrm{~m}^{2} \mathrm{~g}^{-1}$ with a pore size of $13.2 \mathrm{~nm}$. The metal surface area $\left(5.5 \mathrm{~m}^{2} \mathrm{~g}^{-1}\right)$ was measured by hydrogen chemisorption (reproducibility $\pm 0.5 \mathrm{~m}^{2} \mathrm{~g}^{-1}$ ) giving a dispersion of $43 \%$, from which an average metal crystallite size of $2.6 \mathrm{~nm}$ was calculated.

The hydrogenation reactions were performed in a $500 \mathrm{~cm}^{3}$ Büchi autoclave reactor fitted with an oil heating jacket, which could control temperature to $\pm 0.5 \mathrm{~K}$. The reaction temperature was measured in the liquid slurry with accuracy of $\pm 0.1 \mathrm{~K}$ by a sheathed thermocouple. The reactor was equipped with a variable speed stirrer connected to a magnetic drive that could be controlled to $\pm 5 \mathrm{rpm}$. The pressure and gas flow was controlled by a Büchi press-flow gas controller with an accuracy of \pm 0.01 barg and which measured the consumption of hydrogen or deuterium to $0.1 \mathrm{mmol}$. The experimental procedure involved the addition of the catalyst (100 mg Rh/SiO 2 ) and $310 \mathrm{~cm}^{3}$ of 2-propanol (isopropyl alcohol, IPA), which had been degassed, into the reactor before the system was purged with argon. The catalyst was then reduced in situ at $343 \mathrm{~K}$ by sparging hydrogen gas $\left(280 \mathrm{~cm}^{3} \mathrm{~min}^{-1}\right)$ through the mixture for $0.5 \mathrm{~h}$, whilst stirring at $300 \mathrm{rpm}$. The hydrogen gas and stirrer were turned off once reduction was complete and the reactor purged twice with argon and pressurized to 1 barg. The reaction mixture was cooled to the desired temperature (303-343 K) and agitation stopped. Catechol (1,2-dihydroxybenzene, Sigma-Aldrich $\geq 99 \%$ ), resorcinol (1,3-dihydroxybenzene, Sigma-Aldrich 99\%) and hydroquinone (1,4-dihydroxybenzene, Sigma-Aldrich 99\%) were added as mixtures (each reactant was $10 \mathrm{mmol}$ ) to the reactor in $25 \mathrm{~cm}^{3}$ IPA. This was followed by an IPA flush to ensure all reactants entered the reaction mixture, giving a total volume of $350 \mathrm{~cm}^{3}$. The solution was thoroughly mixed by stirring at $300 \mathrm{rpm}$ for 2 min before a sample $\left(2.5 \mathrm{~cm}^{3}\right)$ was withdrawn for analysis. The system was purged with argon before being purged twice with hydrogen before increasing to the desired reaction pressure ( $3 \mathrm{barg}$ ). The reaction was started by setting the stirrer speed to $1000 \mathrm{rpm}$ and the reaction profile followed by withdrawing samples of $2.5 \mathrm{~cm}^{3}$ over a $180 \mathrm{~min}$ for each reaction. For the first 30 min samples were taken every $5 \mathrm{~min}$, this was increased to every 10 and $20 \mathrm{~min}$ for the following 30 and $120 \mathrm{~min}$ respectively. The moles of hydrogen gas consumed during the reaction were also recorded. Mass balance was $100 \pm 2 \%$. The liquid samples were analysed using a Thermo Finnigan Focus gas chromatograph equipped with an FID detector and an AS 3000 autosampler. The column used was an HP-1701 $(30 \mathrm{~m} \times 0.25 \mathrm{~mm} \times 1 \mu \mathrm{m}$ film thickness). Standard tests were performed to ensure the absence of mass transport control.

\section{Results}

\subsection{Hydrogen Reactions}

The competitive hydrogenation of catechol, resorcinol and hydroquinone was investigated with compounds tested in pairs and with all three reactants present (Figs. 1, 2, 3, 4). Individual hydrogenation has been reported elsewhere [6]. The first order rate constants, including those from the individual reactions, are reported in Table 1 .

The competitive reaction of catechol and resorcinol resulted in a significant decrease in the rate of reaction for both isomers as shown in Fig. 1. Slight inhibition of resorcinol hydrogenation occurred with the formation of 3-hydroxycyclohexanone and cis-1,3-cyclohexanediol delayed until 10 and 25 min respectively, whereas catechol reacted immediately. This is in marked contrast to individual hydrogenations where both dihydroxybenzene isomers formed hydrogenated products from the outset. The hydrogenated products from catechol ( $23 \%$ yield) were favoured over those from resorcinol ( $12 \%$ yield).

The competitive hydrogenation of catechol and hydroquinone is shown in Fig. 2. As before, a substantial drop in overall reactivity occurred with both substrates. The inhibition effect observed when resorcinol was reacted with catechol was apparent for hydroquinone when in competition with catechol; both major ring hydrogenated products, 3-hydroxycyclohexanone and cis-1,3-cyclohexanediol, did not form until 10 and $15 \mathrm{~min}$ into the reaction respectively.

Of all pairs of substrates tested competitively, resorcinol and hydroquinone (Fig. 3) showed the greatest decrease in rate of reaction. In reaction with hydroquinone, the conversion of resorcinol after 180 min was only $22 \%$, in comparison resorcinol conversion was $40 \%$ after 180 min, when 
Fig. 1 Competitive reaction of catechol and resorcinol. Conditions: $323 \mathrm{~K}, 3$ barg, $10 \mathrm{mmol}$ of each reactant
Fig. 2 Competitive reaction of catechol and hydroquinone. Conditions: $323 \mathrm{~K}, 3$ barg, $10 \mathrm{mmol}$ of each reactant
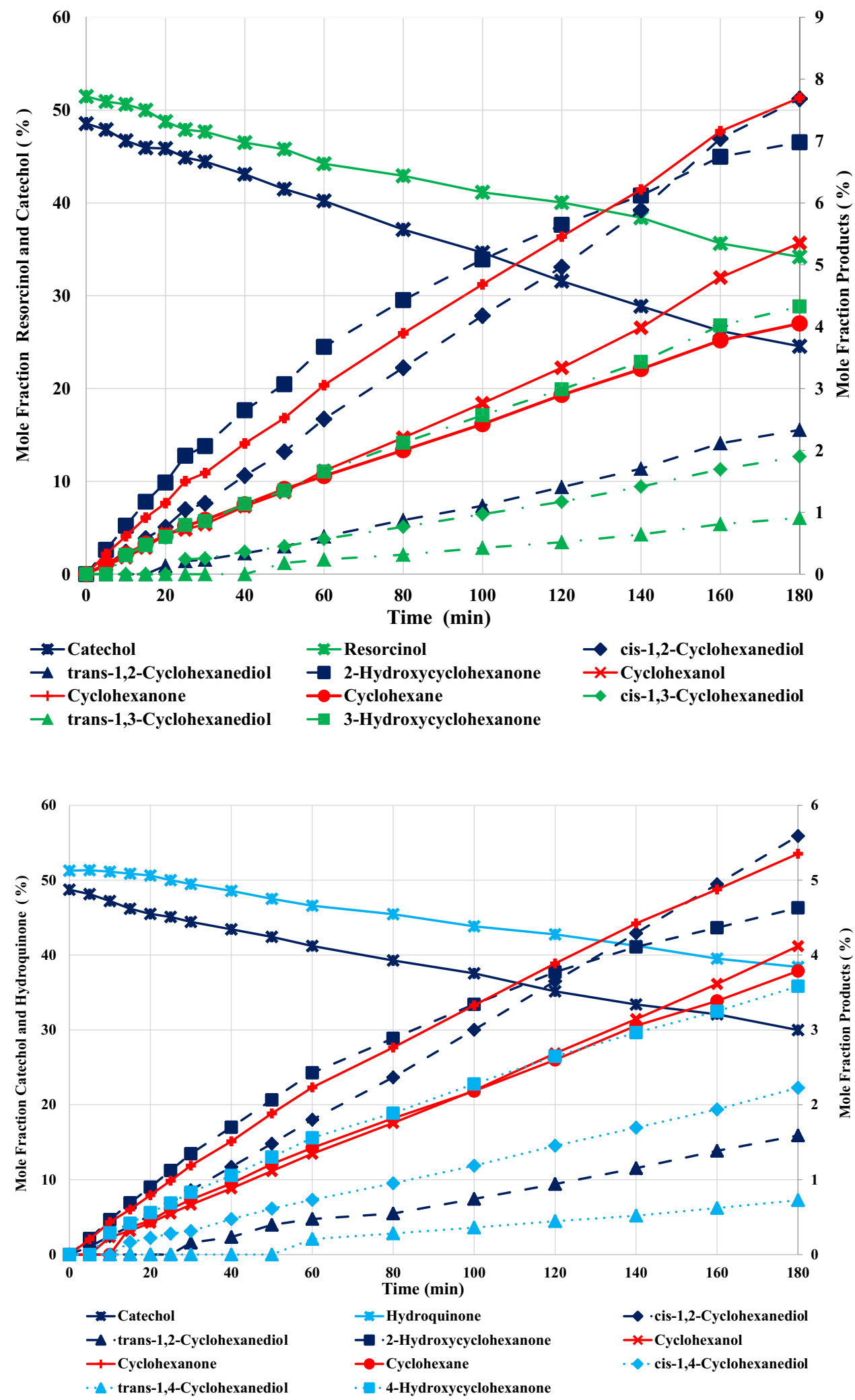

reacted in the presence of catechol. Hydroquinone exhibited a similar decrease in conversion suggesting competition for similar sites on the catalyst between hydroquinone and resorcinol. 
Fig. 3 Competitive reaction of hydroquinone and resorcinol. Conditions: 323 K, 3 barg,

$10 \mathrm{mmol}$ of each reactant
Fig. 4 Comparison of conversion with time for single reactions and the competitive reaction when catechol, hydroquinone and resorcinol were all present. Conditions: $323 \mathrm{~K}, 3$ barg, concentration $10 \mathrm{mmol}$ for each reactant. Individual data calculated from Ref. [6]
Table 1 Competitive

hydrogenation at $323 \mathrm{~K}, 3$ barg hydrogen with $10 \mathrm{mmol}$ of each reactant
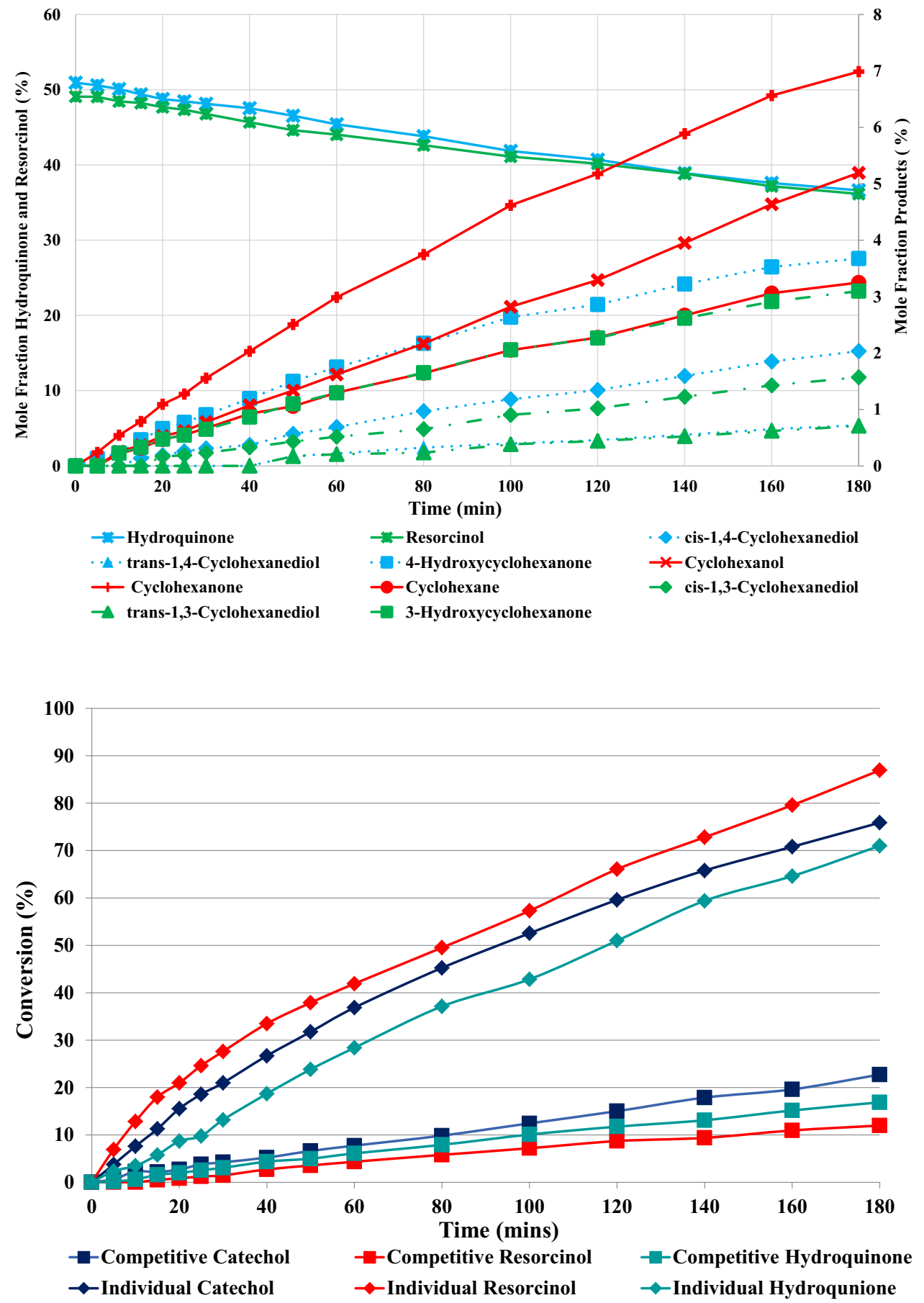

\begin{tabular}{lcccll}
\hline Reactant & \multicolumn{4}{l}{ First order rate constant, $\mathrm{k}\left(\mathrm{min}^{-1}, \times 10^{-3}\right)$} & \begin{tabular}{l} 
Catechol/hyd- \\
\cline { 2 - 6 }
\end{tabular} \\
\cline { 2 - 6 } & Single reactant & $\begin{array}{l}\text { Catechol/ } \\
\text { resorcinol } \\
\text { roquinone } \\
\text { roquinone }\end{array}$ & $\begin{array}{l}\text { Rercinol/hyd- } \\
\text { catechol/resor- } \\
\text { cinol/hydroqui- } \\
\text { none }\end{array}$ \\
\hline Catechol & 8.3 & 5.2 & 6.7 & - & 3.8 \\
Resorcinol & 11.2 & 5.0 & - & 3.5 & 3.5 \\
Hydroquinone & 4.2 & - & 4.8 & 3.8 & 3.0 \\
\hline
\end{tabular}

${ }^{\text {a Data from Ref. [6] }}$ 
A reaction with all three substrates present was carried out and a substantial reduction in rate was observed, as outlined in Fig. 4. The product distribution is shown in Fig. 5. It can be seen from the product yield that cis-1,2-cyclohexanediol and 2-hydroxycyclohexanone, hydrogenated products from catechol, are formed to the greatest extent, emphasising the dominance of catechol in the competitive situation. As before, inhibition of the main hydrogenated products of hydroquinone and resorcinol occurs with the formation of 4-hydroxycyclohexanone and 3-hydroxycyclohexanone delayed until 10 and 40 min respectively.

The ratio of the HDO products was investigated to see if any further information could be gleaned. The data is shown in Table 2. When catechol and resorcinol are reacted together and when catechol and hydroquinone are reacted together, the HDO ratios are typical of that of catechol in the absence of a competing species. When resorcinol and hydroquinone are reacted competitively the HDO product ratio resembles that from hydroquinone, as does the ratio when all three dihydroxybenzene isomers are co-reacted.

\section{Deuterium Reactions}

The competitive reactions were repeated using deuterium instead of hydrogen to try to obtain some extra mechanistic information. The rate constants obtained from the reactions under deuterium, reported in Table 3, were compared with those under hydrogen and a kinetic isotope effect (KIE) calculated. The reaction profiles of the four reactions are shown in Figs. 6, 7, 8 and 9.

The order of reactivity when the three isomers were hydrogenated competitively under hydrogen was catechol $>$ hydroquinone $>$ resorcinol. This order was maintained under deuterium but with catechol $>>$ hydroquinone $>$ resorcinol
Fig. 5 Product distribution from competitive reaction of catechol, resorcinol and hydroquinone. Conditions: $323 \mathrm{~K}$, 3 barg hydrogen and $10 \mathrm{mmol}$ each reactant
Table 2 Ratio of ODH products (cyclohexanone, cyclohexanol and cyclohexane) at $10 \%$ conversion of reactant

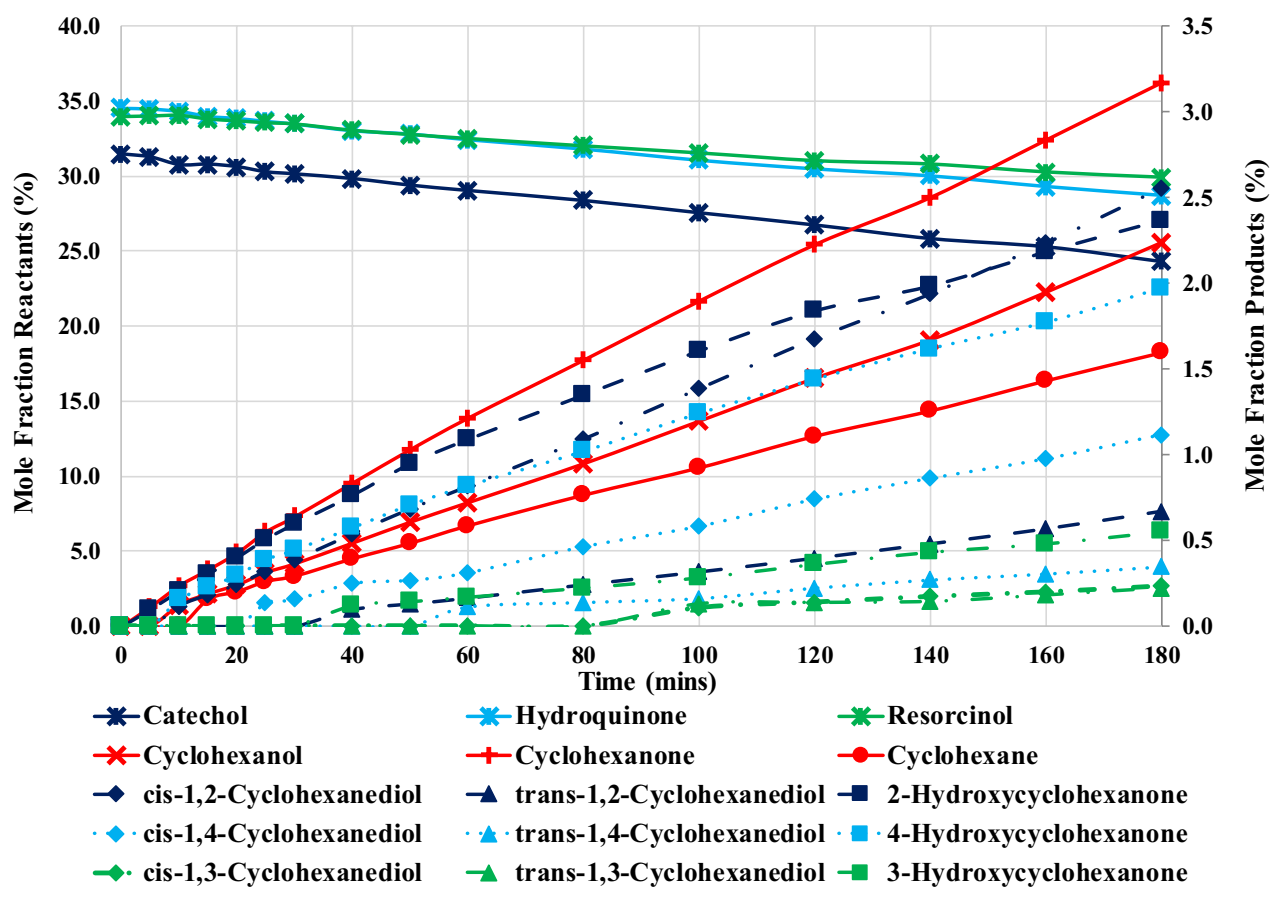

\begin{tabular}{llll}
\hline Reactant & \multicolumn{2}{l}{ Ratio of ODH products } & \\
\cline { 2 - 4 } & Cyclohexanone & Cyclohexanol & Cyclohexane \\
\hline Catechol $^{\mathrm{a}}$ & 1.3 & 1 & 1 \\
Hydroquinone $^{\mathrm{a}}$ & 2.3 & 1.3 & 1 \\
Resorcinol $^{\mathrm{a}}$ & 3.3 & 1.8 & 1 \\
Catechol/resorcinol $_{\text {Catechol/hydroquinone }}$ & 1.9 & 1 & 1 \\
Hydroquinone/resorcinol & 1.6 & 0.9 & 1 \\
Catechol/hydroquinone/resorcinol & 2.4 & 1.3 & 1 \\
\hline
\end{tabular}

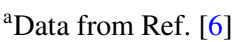


Table 3 First order rate constants for deuterium reactions and kinetic isotope effects (KIEs)

\begin{tabular}{lllll}
\hline Reaction & \multicolumn{2}{l}{ First order rate constant $\left(\times 10^{-3} \mathrm{~min}\right)$} & Kinetic isotope effect, $\mathrm{k}_{\mathrm{H}} / \mathrm{k}_{\mathrm{D}}$ \\
\cline { 2 - 4 } & Catechol & Hydroquinone & Resorcinol & \\
\hline $\mathrm{D}_{2}, \mathrm{C}$ and $\mathrm{R}^{\mathrm{a}}$ & 7.5 & & 3.6 & $\mathrm{C}=0.7 \mathrm{R}=1.4$ \\
$\mathrm{H}_{2}, \mathrm{C}$ and $\mathrm{R}$ & 5.2 & & 5.0 & $\mathrm{C}=1.3 \mathrm{H}=1.2$ \\
$\mathrm{D}_{2}, \mathrm{C}$ and $\mathrm{H}^{\mathrm{a}}$ & 5.9 & 3.9 & & \\
$\mathrm{H}_{2}, \mathrm{C}$ and $\mathrm{H}$ & 7.7 & 4.8 & 3.6 & $\mathrm{H}=1.1 \mathrm{R}=1.0$ \\
$\mathrm{D}_{2}, \mathrm{H}$ and $\mathrm{R}$ & & 3.6 & 3.5 & \\
$\mathrm{H}_{2}, \mathrm{H}$ and $\mathrm{R}$ & & 3.8 & 2.2 & $\mathrm{C}=0.9 \mathrm{H}=1.3 \mathrm{R}=1.4$ \\
$\mathrm{D}_{2}, \mathrm{C}, \mathrm{H}$ and $\mathrm{R}$ & 4.4 & 2.8 & 3.0 & \\
$\mathrm{H}_{2}, \mathrm{C}, \mathrm{H}$ and $\mathrm{R}$ & 3.8 & 3.5 & & \\
\hline
\end{tabular}

${ }^{a} C$ catechol, $R$ resorcinol, $H$ hydroquinone

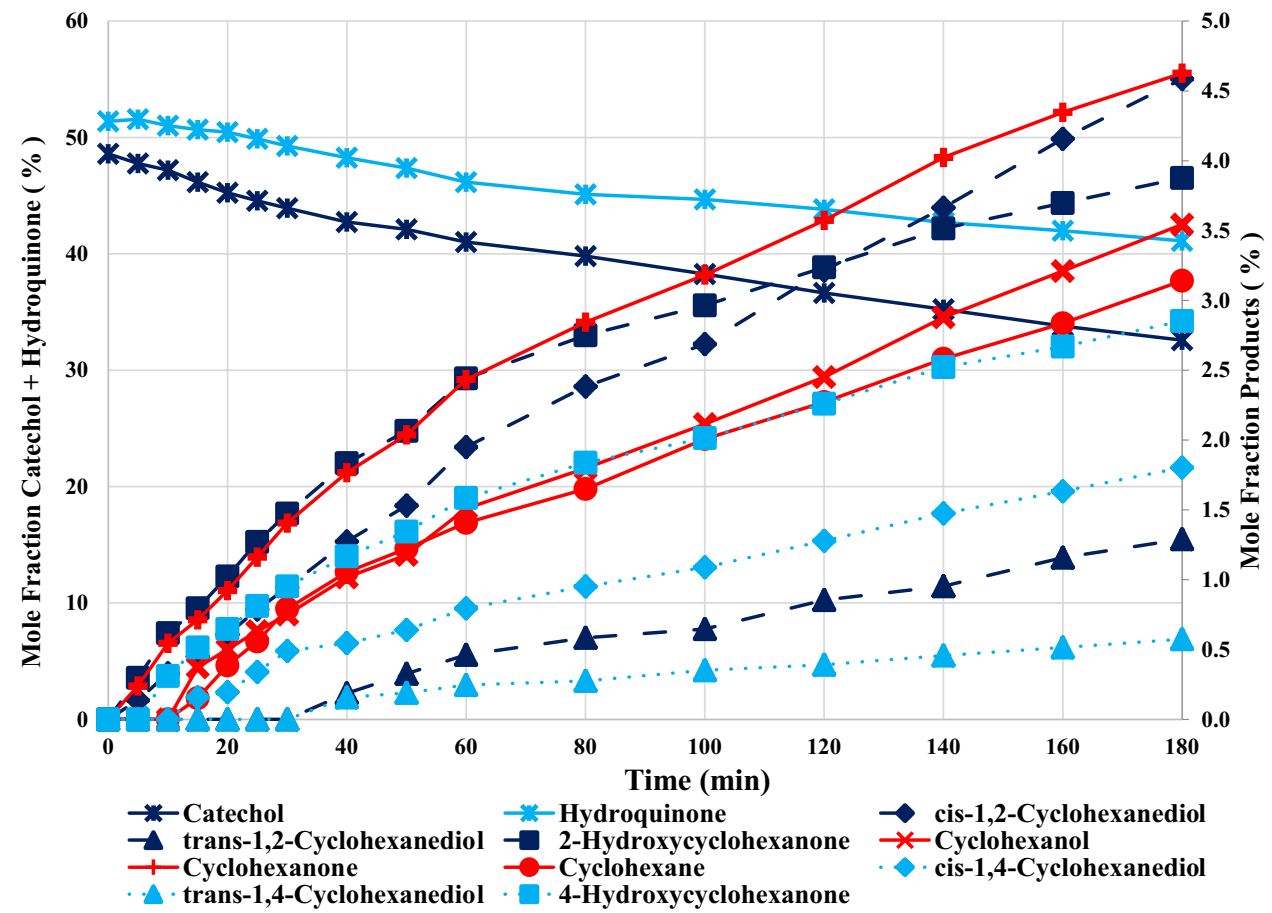

Fig. 6 Competitive reaction of catechol and hydroquinone under $\mathrm{D}_{2}$. Conditions: $323 \mathrm{~K}$, 3 barg $\mathrm{D}_{2}, 10 \mathrm{mmol}$ of each reactant reflecting the inverse KIE for catechol and positive KIEs for hydroquinone and resorcinol.

From Fig. 6 it can be seen that initially the only products are cis-1,2-cyclohexanediol, 2-hydroxycyclohexanone and cyclohexanone, all produced from catechol. After $10 \mathrm{~min}$ 4-hydroxycyclohexanone is formed and by 15 min cyclohexane and cis-1,4-cyclohexanediol are detected. It takes $40 \mathrm{~min}$ before trans-isomers are formed. Both catechol and hydroquinone exhibit positive KIEs with the rate under hydrogen faster than that under deuterium.

In the competitive reaction of catechol and resorcinol shown in Fig. 7, the initial products are 2-hydroxycyclohexanone and 3-hydroxycyclohexanone followed by cis-1,2-cyclohexanol. Subsequently trans-1,2-cyclohexanol, cis-1,3-cyclohexanol and cyclohexanone are all produced. However, it is $30 \mathrm{~min}$ before cyclohexane is detected and 40 min before trans-1,3-cyclohexanol is seen. This is a significant change from the behaviour under hydrogen.

The competitive reaction of hydroquinone and resorcinol under deuterium (Fig. 8) reveals that the initial products were 4-hydroxycyclohexanone and cyclohexanone, both formed from hydroquinone. Subsequent products were cyclohexanol and 3-hydroxycyclohexanone, cis-1,4-cyclohexandiol was not detected until $15 \mathrm{~min}$ and it was $20 \mathrm{~min}$ before cyclohexane and cis-1,3-cyclohexandiol were observed.

Figure 9 shows the effect of reacting all three dihydroxybenzenes simultaneously. The initial product was cyclohexanone followed by cis-1,2-dihydroxycyclohexanol and 2-hydroxycyclohexanone from catechol. Subsequently 
Fig. 7 Competitive reaction of catechol and resorcinol under $\mathrm{D}_{2}$. Conditions: $323 \mathrm{~K}, 3$ barg $\mathrm{D}_{2}, 10 \mathrm{mmol}$ of each reactant

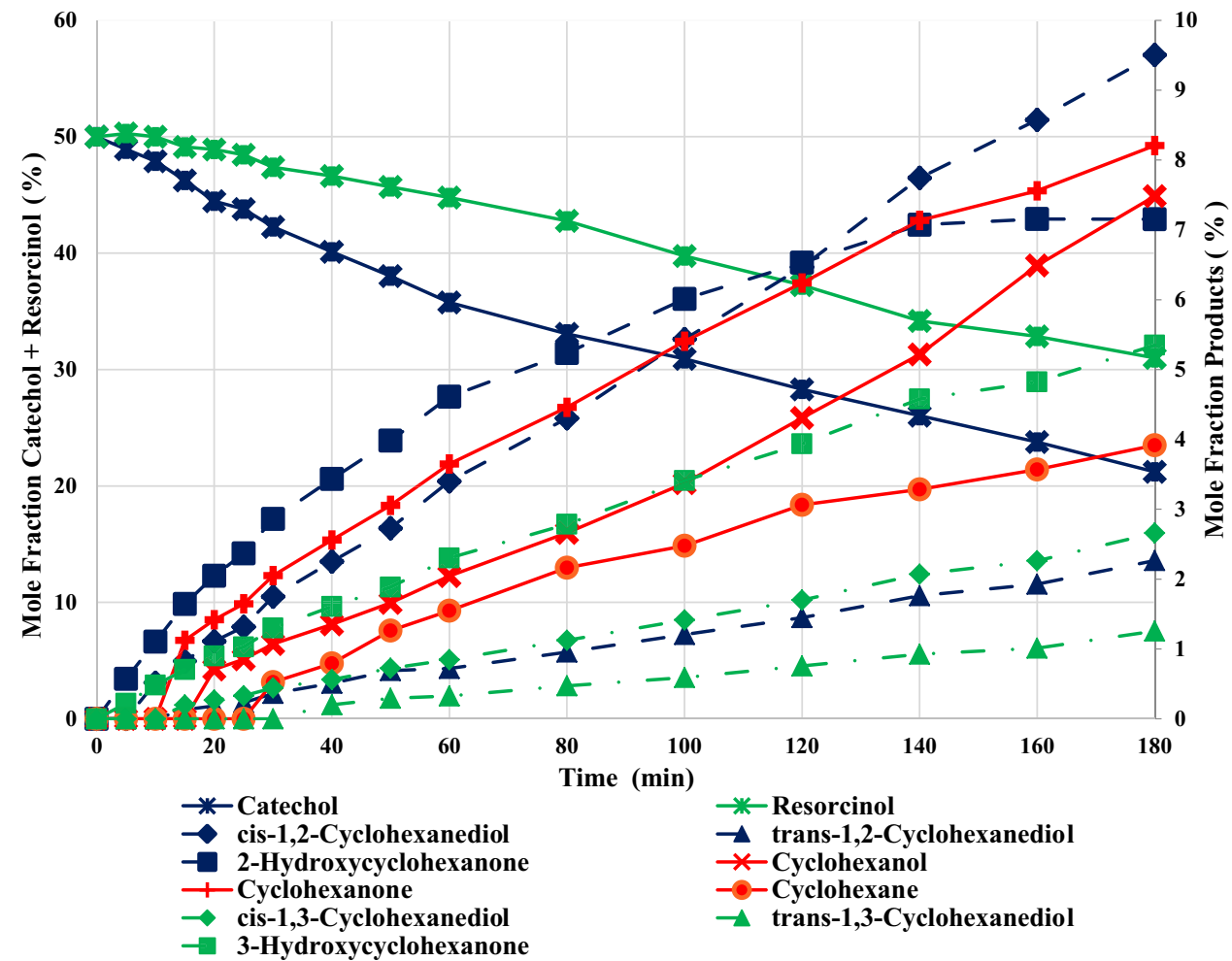

Fig. 8 Competitive reaction of hydroquinone and resorcinol under $\mathrm{D}_{2}$. Conditions: $323 \mathrm{~K}$, 3 barg $D_{2}, 10 \mathrm{mmol}$ of each reactant

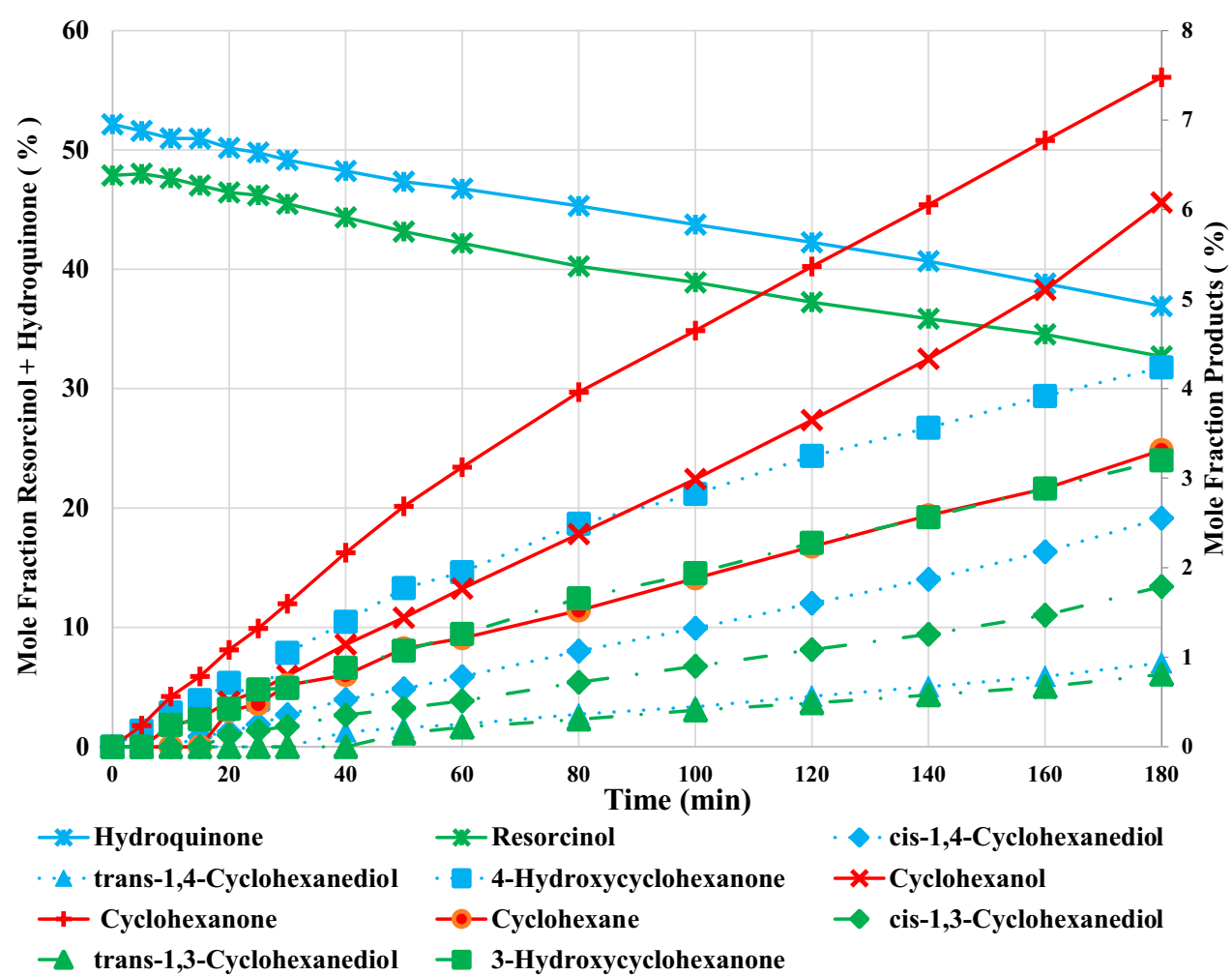


Fig. 9 Competitive reaction of catechol, hydroquinone and resorcinol under $\mathrm{D}_{2}$. Conditions: $323 \mathrm{~K}, 3$ barg $\mathrm{D}_{2}, 10 \mathrm{mmol}$ of each reactant

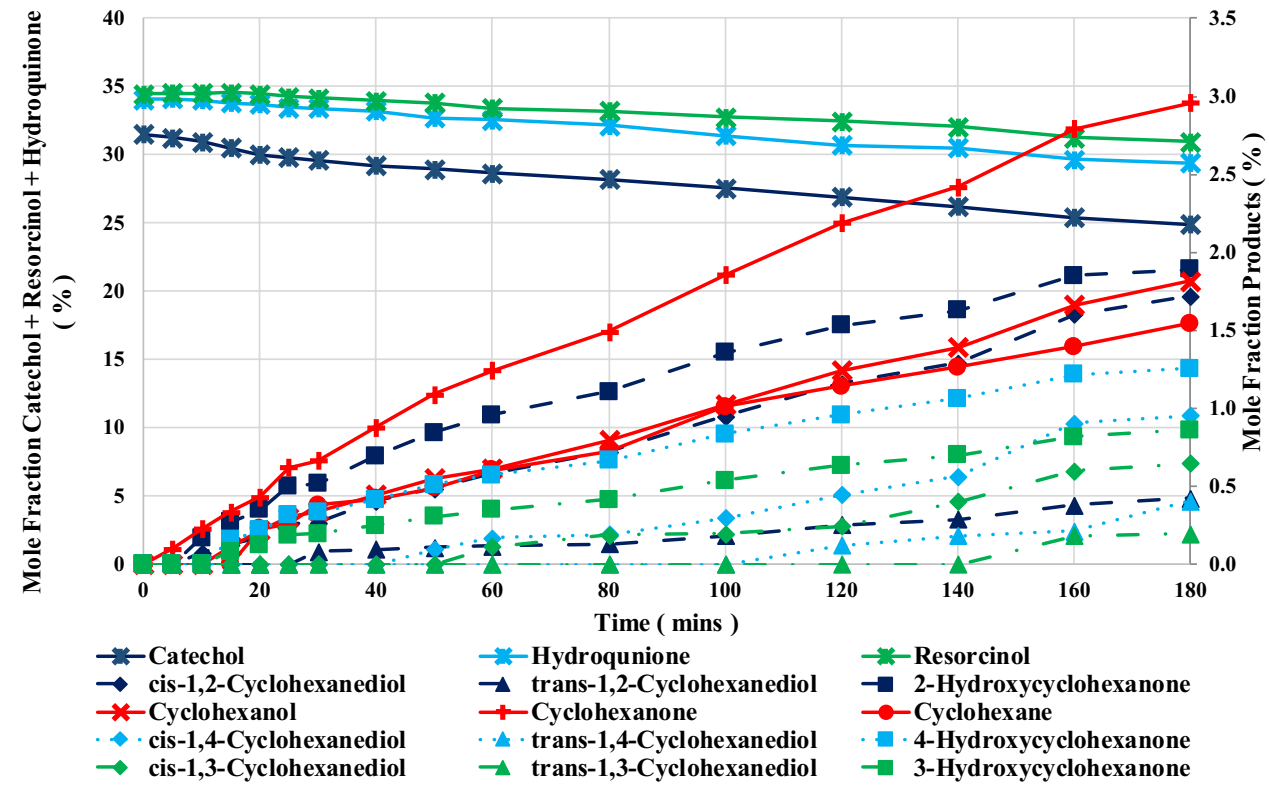

3- and 4-hydroxycyclohexanone were produced as was cyclohexanol, however it was $120 \mathrm{~min}$ before trans-1,4-cyclohexanol was detected and 160 min before trans-1,3-cyclohexanol was observed.

\section{Discussion}

To aid the discussion a reaction scheme for hydroquinone is presented in Fig. 10 (a similar scheme would apply to catechol and resorcinol). The competitive hydrogenation of catechol and resorcinol shows, as expected, a decrease in rate for both reactants relative to their single hydrogenation. However, what was not expected was that the rate of reaction of resorcinol would be less that catechol. In single reactant tests, resorcinol was the most reactive of the three isomers [6]. Nevertheless, a similar result was observed with xylene isomers [16]. Meta-xylene was more reactive than ortho-xylene in single reagent tests but in a competitive environment ortho-xylene was much more active. This behaviour with the xylenes was interpreted in terms of strength of adsorption and a similar interpretation, with catechol more strongly adsorbed than resorcinol, could apply here. In the initial stages of reaction only
Fig. 10 Reaction scheme for hydroquinone. Products in red were not detected by GC. Similar schemes apply to catechol and resorcinol

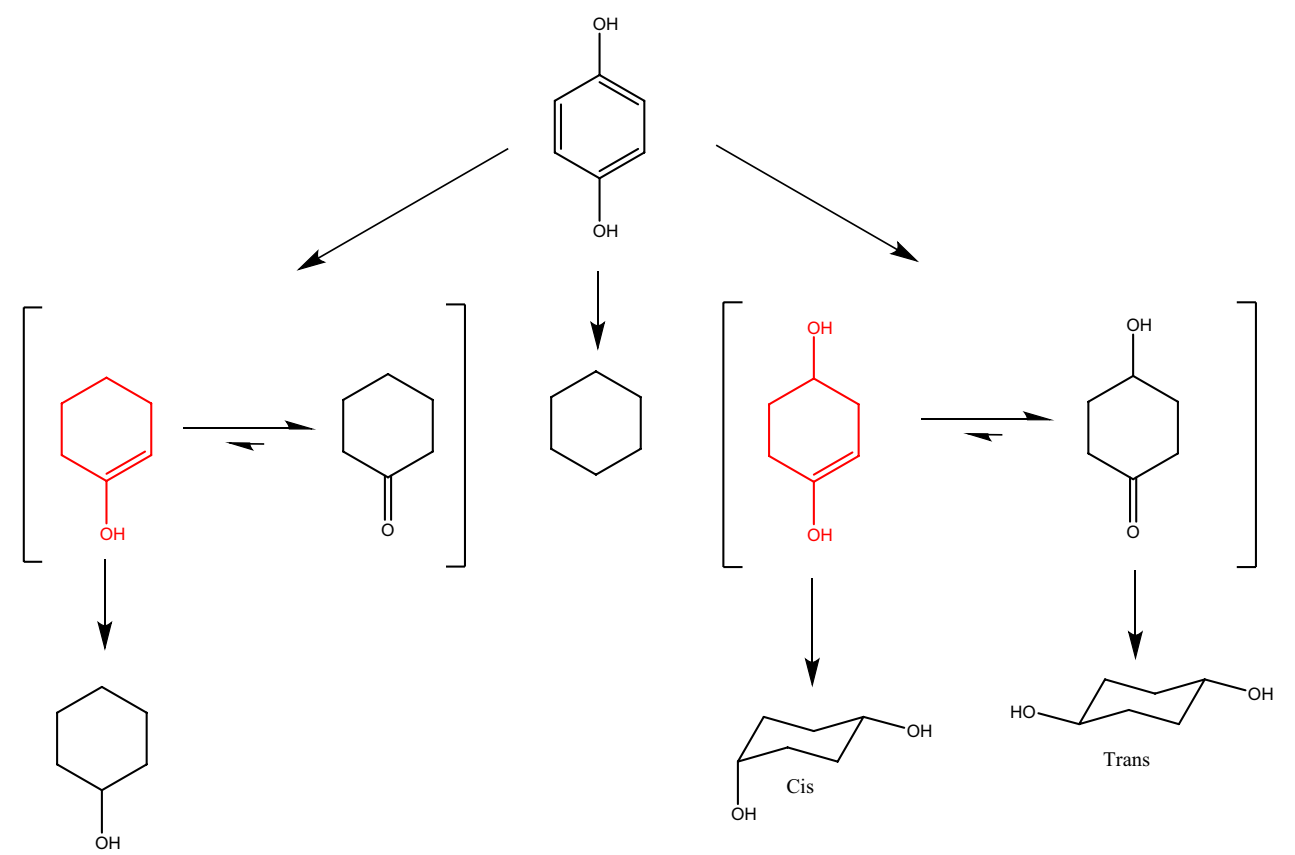


cyclohexane, cyclohexanol and cyclohexanone as well as 2-hydroxycyclohexanone and cis-1,2-cyclohexandiol are formed: no 1,3-isomer products are detected. Nevertheless, mass balance analysis indicates that $83 \%$ of initial HDO products come from resorcinol, with only $17 \%$ from catechol. This is in keeping with the individual reactions, where resorcinol produced more HDO products than catechol. However the ratio of cyclohexanone: cyclohexanol: cyclohexane from the competitive reaction, reported in Table 2, reflects the ratio observed from catechol rather than resorcinol; indicating that resorcinol HDO selectivity has been altered by the presence of catechol. Nevertheless, in the competitive reaction, HDO products are the primary products from resorcinol. Therefore, in the early stages of the competitive reaction, resorcinol can adsorb in the presence of catechol and undergo HDO but not discrete hydrogenation. This suggests that there are sites for HDO and sites for hydrogenation. It is only subsequently that production of 3-hydroxycyclohexanone is observed. It has been proposed that the formation of cis-1,3-cyclohexandiol and trans-1,3-cyclohexandiol is from 1,3-dihydroxycyclohex-1-ene and 3-hydroxycyclohexanone respectively with 3-hydroxycyclohexanone hydrogenation involving desorption and re-adsorption [6] to account for the delay in production of the trans-isomer. However, in the competitive environment, the production of both cis- and trans1,3-isomers was delayed suggesting that in the competitive system, hydrogenation of 1,3-dihydroxycyclohex-1-ene was initially slow relative to isomerisation to 3-hydroxycyclohexanone and desorption. A similar pattern was observed between catechol and hydroquinone suggesting analogous chemistry with catechol being more strongly bound than hydroquinone on hydrogenation sites. This would be in keeping with the xylene study [16].

When hydroquinone and resorcinol were hydrogenated in competition to each other there was a marked change in the product distribution in that the hydrogenolysis products were significantly favoured over the hydrogenated products. Indeed, cyclohexanone and cyclohexanol were the major products of the reaction. This is in keeping with the reactions of the DHB isomers in a non-competitive environment but hydrogenolysis was enhanced in the competitive medium. In the initial stages of reaction, hydroquinone produced 1,4-hydroxycyclohexanone and $88 \%$ of cyclohexanone. As the reaction progressed the amount of HDO products produced by each DHB isomer changed such that initially it was 7:1 in favour of hydroquinone over resorcinol but by the end of the reaction the ratio was 1:1, with each isomer producing equal amounts of HDO products. The selectivity to HDO products was $\sim 63 \%$. Under these conditions we have shown that cyclohexane, cyclohexanone and cyclohexanol are primary products. Cyclohexanone can be hydrogenated to cyclohexanol but only once more strongly bound aromatic species have been hydrogenated [11]. At the end of the reaction conversion of hydroquinone and resorcinol is $\sim 26 \%$ respectively and the overall yield of HDO products is $~ 32 \%$, when this is compared with the HDO product yield at $26 \%$ conversion for the individual DHB isomer reactions the yield for each of them was $~ 15 \%$ giving a combined yield of $30 \%$ [6]. These comparisons suggest that the presence of the second reactant has no significant effect on the HDO reaction and that the only effect of the second component is to reduce the rate of hydrogenation. This is in keeping with the literature where the hydrogenation functionality was significantly reduced in a competitive environment $[14,15]$.

When the three dihydroxybenzene isomers were reacted together, cyclohexanone and 2-hydroxycyclohexanone were the initial products, both produced from catechol. Subsequently cyclohexanol, cis-1,2-cyclohexanol and 4-hydroxycyclohexanone were produced. At this stage $86 \%$ of the HDO products were produced from catechol and $14 \%$ from hydroquinone. After 15 min cyclohexane is produced and by then $47 \%$ of the HDO products are produced from hydroquinone, $28 \%$ from catechol and $25 \%$ from resorcinol. Note that resorcinol has produced no hydrogenated products at this stage. Therefore, even though catechol is more strongly bound and gives rise to the initial products, hydroquinone and resorcinol favour HDO relative to catechol HDO and hydrogenation.

In the initial stages of the reaction with all three isomers, only catechol reacted producing the two ketones cyclohexanone and 2-hydroxycyclohexanone, in both cases hydrogenation to the respective alcohol was a secondary step requiring re-adsorption. The difference in timescale between the production of the cis-1.2-cyclohexanol and trans-1,2-cyclohexanol was related to the hydrogenation of the different intermediates, with the cis-isomer being produced from hydrogenation of 1,2-dihydroxycyclohex1-ene, while trans-1,2-cyclohexanol was produced from 2-hydroxycyclohexanone [6]. Similar behaviour was seen with hydroquinone when it initiated reaction after $10 \mathrm{~min}$. Resorcinol was significantly inhibited requiring $40 \mathrm{~min}$ before 3-hydroxycyclohexanone was detected and another hour before 1,3-alcohols were observed. Along with the significant inhibition, the cis/trans ratio of the 1,3-cyclohexanols was unusual in that both isomers were simultaneously produced with $\mathrm{a} \sim 1: 1$ ratio. This behaviour was not observed individually with resorcinol [6] or with either of the pair reactions involving resorcinol so was unique to the reaction with the three isomers present. Note that the cis/trans ratios for the 1,2- and 1,4-isomers were not noticeably affected. This suggests that the hydrogenation mechanism for at least one of the 1,3-cyclohexandiol isomers was being influenced by the combination of other species on the surface.

To further help with understanding the processes occurring the reactions were all repeated using deuterium instead 
of hydrogen. When catechol and resorcinol were hydrogenated using deuterium, catechol displayed an inverse kinetic isotope effect (iKIE) similar to that found when catechol was reacted singly. In contrast resorcinol displayed a normal KIE. This was surprising because individually resorcinol had displayed an iKIE [6]. The change to a normal KIE for resorcinol implies a significant change in surface energetics for resorcinol ring hydrogenation, which is consistent with hydrogenation being affected by the presence of a second reactant $[14,15]$. The iKIE was explained by the change from $\mathrm{sp}^{2}$ hybridized carbon to $\mathrm{sp}^{3}$ hydridized carbon being the rate determining step [16], however the normal KIE suggests that hydrogen bond breaking or making is involved in the rate determining step. Similar behaviour was observed with competitive deuteration of catechol and hydroquinone in that a positive KIE of 1.3 for catechol and 1.2 for hydroquinone was calculated. Again, one reactant has significantly changed its KIE, with catechol going from 0.7 to 1.3 whereas for hydroquinone the KIE remained constant. When hydroquinone and resorcinol were hydrogenated using deuterium neither exhibited a KIE with the rate in hydrogen and deuterium almost identical. The three pair experiments using deuterium revealed that the surface energetics changed with each combination as observed by the changes in the kinetic isotope effect data. When all three isomers were reacted together using deuterium in place of hydrogen the rate of catechol hydrogenation increased whereas hydrogenation of resorcinol and hydroquinone decreased such that catechol exhibited an iKIE, while hydroquinone and resorcinol exhibited a positive KIE. These results indicate that the presence of competing reactants has a dramatic effect on the energetics of the hydrogenation reactions of the individual components. However, no obvious trend was discernible and changes detected from pair reactions were not carried over to the triplet test. One possible explanation would be a rearrangement of the surface due to the liberation of the heat of adsorption [17, 18], which would change depending on which isomers were adsorbed. This would imply the surface was metastable as outlined by Spencer [19].

Another significant change observed when deuterium was used was the delay in production of the hydrogenolysis products, when catechol and resorcinol were reacted as a pair. In the hydrogen reaction all three HDO products (cyclohexanone, cyclohexanol and cyclohexane) were all observed initially, whereas under deuterium the initial products were 2-hydroxycyclohexanone and 3-hydroxycyclohexanone. The HDO products were each observed at a different time (15 min for cyclohexanone, $20 \mathrm{~min}$ for cyclohexanol and $30 \mathrm{~min}$ for cyclohexane). This behaviour was similar to that observed with individual deuteration of catechol and resorcinol [6], so is not an effect of the competitive reaction per se, although the delay is lengthened. The cause of the delay in the single reactions was postulated to be due to deuterium blocking HDO sites on the rhodium crystallite [6] however, in the single reactant systems cyclohexane was clearly inhibited but it was less clear for cyclohexanone and cyclohexanol. In the competitive system the delay for each product was much more definitive. This then casts doubt on the deuterium blocking proposal as it would require three sites, one for each HDO product. From the analysis of the HDO products in the competitive reactions is seems more likely that there is a single HDO site but that the selectivity between cyclohexane, cyclohexanone and cyclohexanol is related to the stability of the reaction intermediates produced from each dihydroxybenzene. Mechanistically adsorption through the ring would be the most likely for hydrogenation whereas the initial step for HDO may come via bonding to the surface by an $-\mathrm{OH}$ group.

\section{Conclusions}

The competitive hydrogenation and HDO of dihydroxybenzene isomers was investigated over a $\mathrm{Rh} /$ silica catalyst at $323 \mathrm{~K}$ and 3 barg hydrogen. In general, the rate of reaction was less than that observed when no competitive reactant was present. Catechol initially inhibited resorcinol and hydroquinone hydrogenation but not HDO suggesting separate sites for hydrogenation and HDO. When resorcinol and hydroquinone were competitively reacted HDO became the favoured reaction. The data suggested that cyclohexane and cyclohexanone were primary products, with the ratio of HDO products produced at low dihydroxybenzene conversion dependent upon the DHB isomer (cyclohexanol may be a primary product but in the later stages of the reaction cyclohexanone was converted to cyclohexanol). When all three DHB isomers were reacted together, initially $86 \%$ of the HDO yield came from catechol with the rest from hydroquinone. Even when resorcinol finally reacted, it produced HDO products first. Deuterium was used and the complexity of the reaction energetics were revealed. When reacted in pairs usually one of the pair would show a change in KIE from that observed when reacted in isolation. For example, resorcinol displayed a positive KIE when reacted alone but displayed an iKIE when reacted with catechol, whereas when catechol and hydroquinone were co-reacted, catechol exhibited a positive KIE in contrast to the iKIE observed when reacted alone or with resorcinol. These results indicate that the presence of competing reactants had a dramatic effect on the energetics of the hydrogenation reactions of the individual components, however no obvious trend was discernible. Using deuterium also had a significant effect on HDO energetics, with reactions significantly inhibited, reinforcing the view that hydrogenation and HDO are mechanistically 
separate. This effect on reaction energetics observed when more than one substrate was present, highlights the limitations of studying one single model compound as a route to understanding the processes required for the upgrading of a true bio-oil feed.

Acknowledgements The authors would like to thank Innospec Ltd. and University of Glasgow for funding for one of us (KK).

Open Access This article is licensed under a Creative Commons Attribution 4.0 International License, which permits use, sharing, adaptation, distribution and reproduction in any medium or format, as long as you give appropriate credit to the original author(s) and the source, provide a link to the Creative Commons licence, and indicate if changes were made. The images or other third party material in this article are included in the article's Creative Commons licence, unless indicated otherwise in a credit line to the material. If material is not included in the article's Creative Commons licence and your intended use is not permitted by statutory regulation or exceeds the permitted use, you will need to obtain permission directly from the copyright holder. To view a copy of this licence, visit http://creativecommons.org/licenses/by/4.0/.

\section{References}

1. Saidi M, Samimi F, Karimipourfard D, Nimmanwudipong T, Gates BC, Rahimpour MR (2014) Energy Environ Sci 7:103-129

2. Mäki-Arvela P, Murzin DY (2017) Catalysts 7:265

3. Bouxin FP, Zhang X, Kings IN, Lee AF, Simmons MJH, Wilson K, Jackson SD (2017) Appl Catal A 539:29-37

4. Fócil AAG, Fócil SG, Sotelo VMC, Grimm RL, García FG, Santiago ER, Vargas CES, Ramírez MAV, Heredia JAR (2019) Energy Technol 7:1801112
5. McVeigh A, Bouxin FP, Jarvis MC, Jackson SD (2016) Catal Sci Technol 6:4142-4150

6. Kirkwood K, Jackson SD (2020) Catalysts 10:584

7. Mohammadian Z, Peyrovi MH, Parsafard N (2019) Chem Phys Lett 715:367-374

8. Canning AS, Jackson SD, Monaghan A, Wright T (2006) Catal Today 116:22-29

9. Hamilton CA, Jackson SD, Kelly GJ, Spence R, de Bruin D (2002) Appl Catal A 237:201-209

10. Balázsik K, Szôri K, Szőllősi G, Bartók M (2011) Chem Commun 47:1551-1552

11. Alshehri F, Feral C, Kirkwood K, Jackson SD (2019) React Kinet Mech Catal 128:23-40

12. Xie L-F, Xu Y-P, Shi X-L, Wang F, Duan P-G, Li SC (2020) Catal Today 355:65-74

13. Ryymin E-M, Honkela ML, Viljava TR, Krause AOI (2011) Appl Catal A 389:114-121

14. Shu R, Lin B, Zhang J, Wang C, Yang Z, Chen Y (2019) Fuel Proc Tech 184:12-18

15. Lin B, Li R, Shu R, Wang C, Yuan Z, Liu Y, Chen Y (2020) J Energy Inst 93:847-856

16. Alshehri F, Weinert HM, Jackson SD (2017) React Kinet Mech Catal 122:699-714

17. Sun G, Fuller JT III, Alexandrova AN, Sautet P (2021) ACS Catal 11:1877-1885

18. Gronbeck H, Tomainek D, Kim SG, Rosen A (1997) Chem Phys Lett 264:39-43

19. Spencer MS (1986) Nature 323:685-687

Publisher's Note Springer Nature remains neutral with regard to jurisdictional claims in published maps and institutional affiliations. 